\title{
DISTRIBUTED SECURE COORDINATED CONTROL FOR MULTIAGENT SYSTEM
}

\author{
Miss. Kadam R. M. \\ Department of CSE \\ AITRC, Vita, Maharashtra, India
}

\author{
Miss.Kadam M.S. \\ Department of CSE \\ AITRC, Vita, Maharashtra, India
}

\author{
Mr. Jadhav D. K \\ Department of CSE \\ AITRC, Vita, Maharashtra, India
}

\begin{abstract}
The multiagent system studies the problem in a system with random delays governed by a Markov chain. The communication topology is assumed to be directed and fixed. An agent is a software entity that is situated in systems environment and is capable of flexible, autonomous action in order to meet its design objectives. Agent is a software which detects the abnormal behavior of files, folders and software's and also scan system for various attacks like man in middle attack, denial of services and data alteration. After finding the viruses as well as attacks made by unknown resources it give permission to authorized user to block that resource or prevent system form that attacks by applying specified rules.
\end{abstract}

\section{INTRODUCTION}

Recent years have witnessed an increasing attention on distributed cooperative control ofreal-world multiagent systems due to its widespread applications in various fields such as distributed control of team robots, design of sensor networks, formation control of vehicles, rendezvous of mobile agents, and synchronization of coupled chaotic oscillators. A fundamental yet interesting issue on this topic is to develop distributed controllers using only relative local information such that as time goes on, all the agents eventually achieve state consensus of the whole group. As an effective consensus seeking approach, consensus tracking problem has been widely studied for linear multiagent systems. Distributed secure coordinated control of multiagent systems is an interesting and important problem. Multiagent systems, like all large-scale spatially distributed systems, are vulnerable to cyber-attacks due to the development of network information and communication technologies.

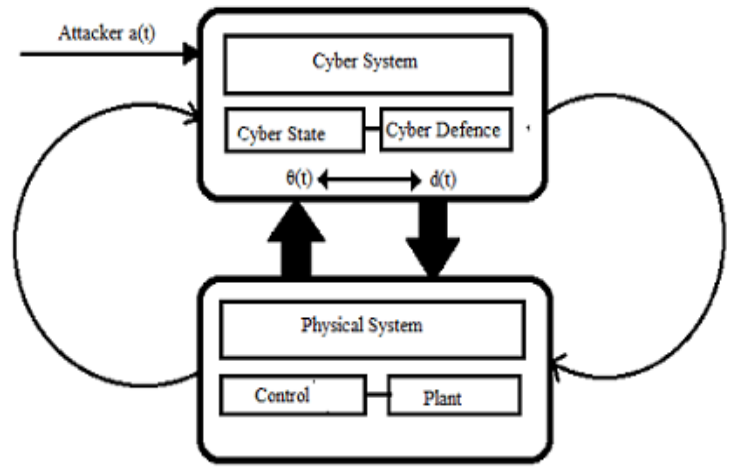

Fig. 1. System Architecture

In a networked multi-agent system, an interaction between the physical space and cyber space is captured by their dynamics. a hybrid system with continuous-time and discrete-time states. The multi-agent physical system state $\mathrm{xi}(\mathrm{t})$ is controlled by a distributed secure controller ui(t). In cyber space under attacks modeled by a random Markov jump process, the cyber system state $\theta(\mathrm{t})$ is controlled by a cyber defense mechanism $d$ used by the network administrator as well as the attacker's action a. The attacker intends to remove the connection edges in a networked multiagent system by launching strategic attacks on graphs, which results in all kinds of new possible graph topologies. These topologies are paralyzed when the graph connectivity is broken. A distributed resilient control algorithm will be developed such that the network would not lose the secure consensus tracking performance. The hybrid nature Fig. 1. Hybrid stochastic secure control framework in a multiagent system. of the multiagent system leads to the adoption of a class of hybrid systems model. Therefore, a framework of this hybrid stochastic secure control for a networked multiagent system is illustrated in Fig. 1. 


\section{International Journal of Engineering Applied Sciences and Technology, 2019 \\ Vol. 3, Issue 12, ISSN No. 2455-2143, Pages 103-106 \\ Published Online April 2019 in IJEAST (http://www.ijeast.com)}

\section{PROPOSED ALGORITHM}

A. RSA algorithm -

RSA involves a public key and private key. The public key can be known to everyone; it is used to encrypt messages. Messages encrypted using the public key can only be decrypted with the private key. The keys for the RSA algorithm are generated the following way:

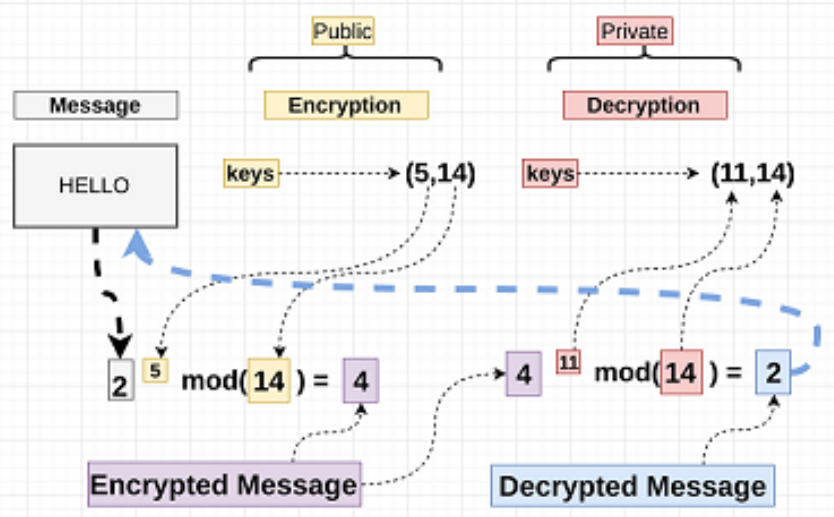

Fig. 2. DWT Decomposition model

1. Choose two different large random prime numbers $\mathrm{p}$ and $\mathrm{q}$

2. Calculate $\mathrm{n}=\mathrm{pq}$

- $\quad \mathrm{N}$ is the modulus for the public key and the private keys

3. Calculate the totient: $\phi=(\mathrm{p}-1)(\mathrm{q}-1)$.

4. Compute the secret exponent $\mathrm{d}, 1<\mathrm{d}<\phi$, such that ed $\equiv 1 \bmod \phi$.

5. The public key is (n,e) and the private key (d,p,q). Keep all the values $d, p, q$ and $\phi$ secret. [Sometimes the private key is written as $(n, d)$ because you need the value of $n$ when using $d$. Other times we might write the key pair as $((\mathrm{N}, \mathrm{e}), \mathrm{d})$.]

The operation of channel separation is applied on the watermarked color image to generate its sub images, and then 2-level discrete wavelet transform is applied on the sub images to generate the approximate coefficients and detail coefficients.

\section{$B$. Analysis of System Stability-}

We propose a new approach which is a System stability for Multiagent System over the different attacks like Denial of Service, Data Alteration and Man of middle attack. There is different methods are available like Laplacian based matrix method and the Lyapunov's method.

\section{EXPERIMENT AND RESULT}

The result set for this evaluation experiment file randomly selected from the machine. Eclipse software platform is use to perform the experiment. The PC for experiment is equipped with an Intel I3 $2.4 \mathrm{GHz}$ laptop and $2 \mathrm{~GB}$ memory storage.

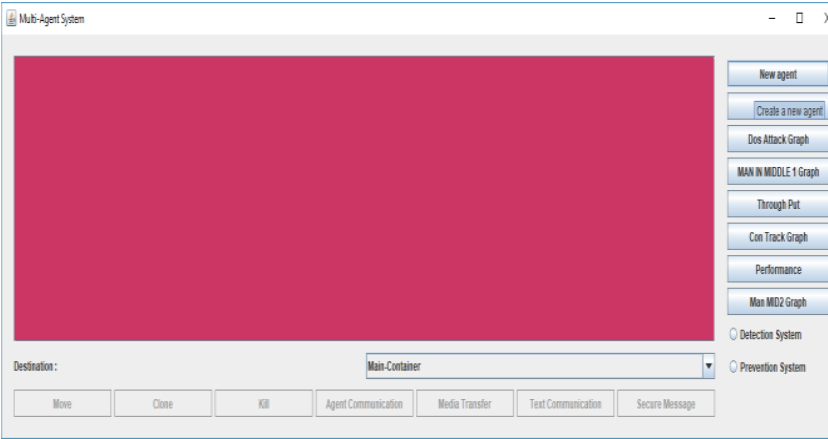

(a)

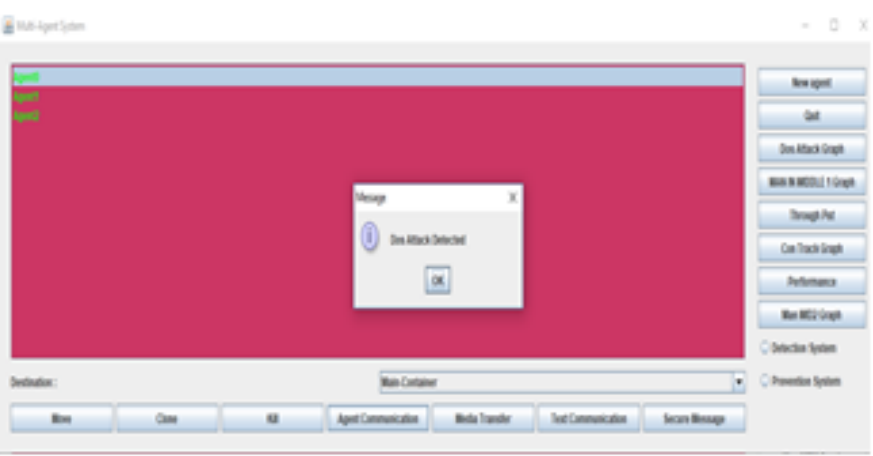

(b)

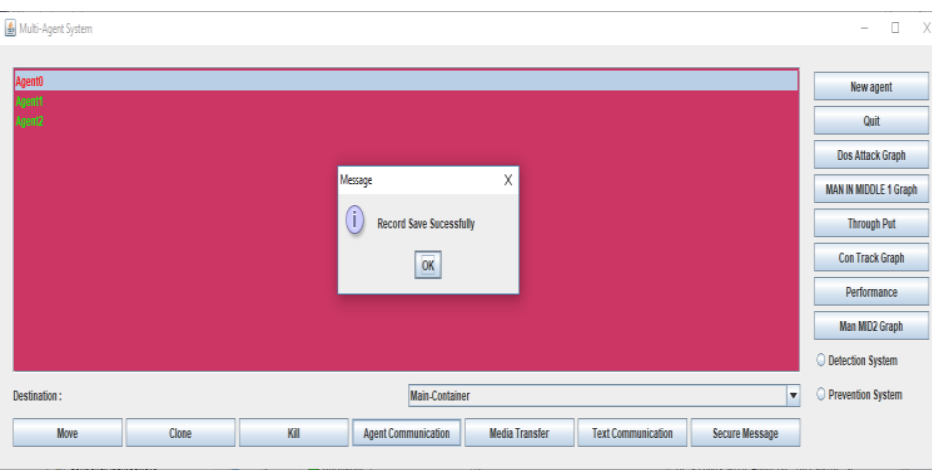

(c) 
International Journal of Engineering Applied Sciences and Technology, 2019

Vol. 3, Issue 12, ISSN No. 2455-2143, Pages 103-106

Published Online April 2019 in IJEAST (http://www.ijeast.com)

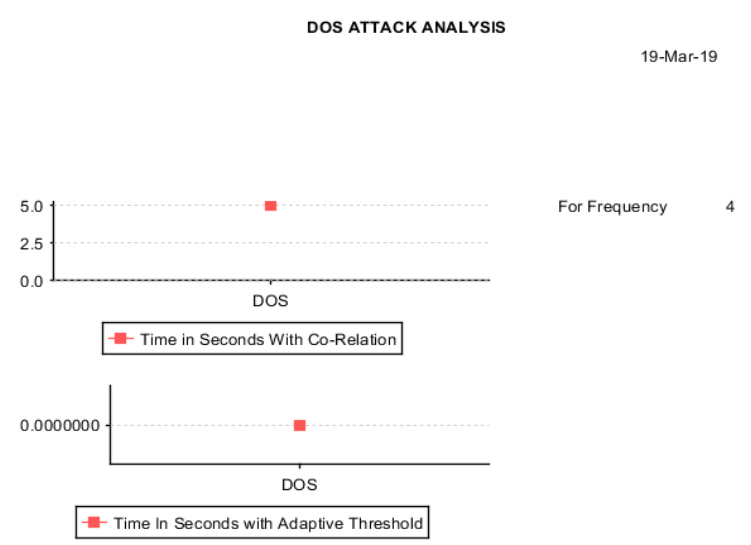

(d)

Fig. 3. (a) Frame work (b) DOS Attack Detection (c) Record saved (d) DOS Attack Analysis

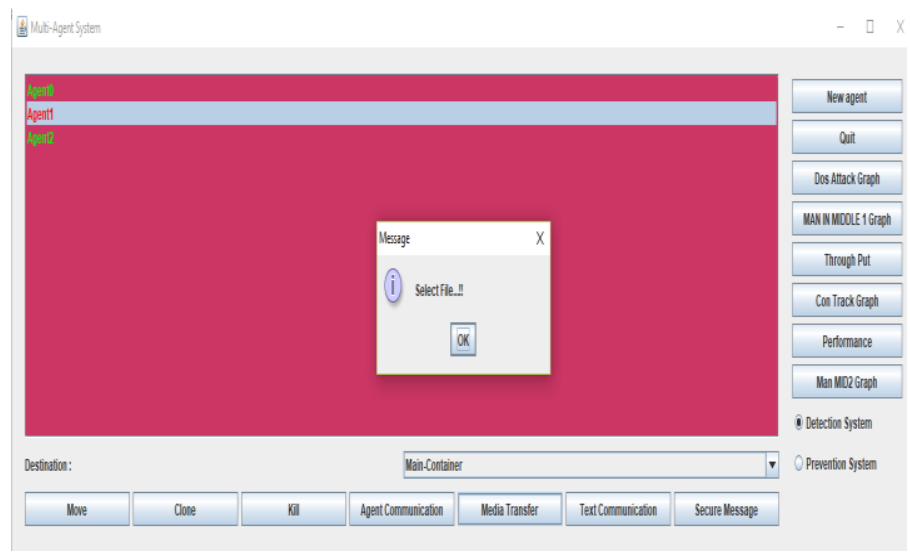

(a)

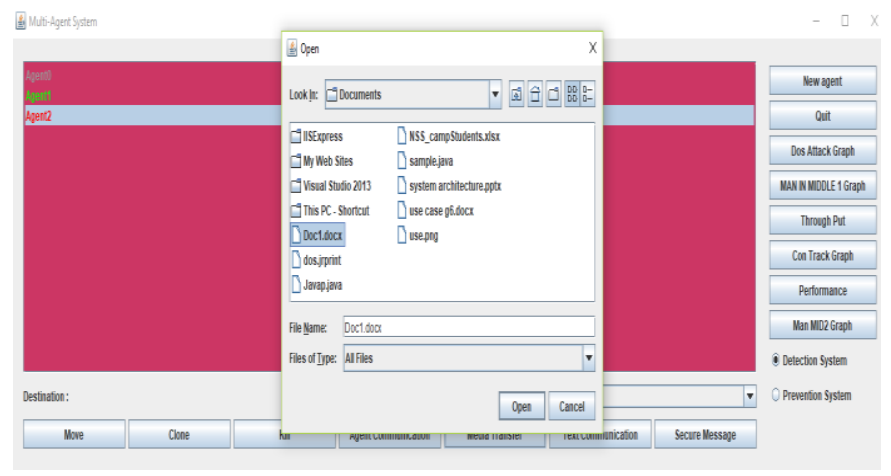

(b)

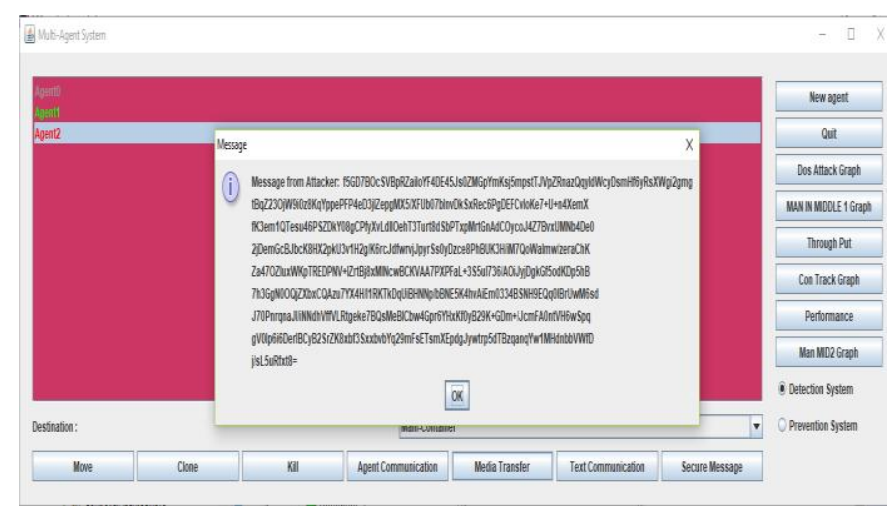

(c)

Data Alteration ATTACK ANALYSIS

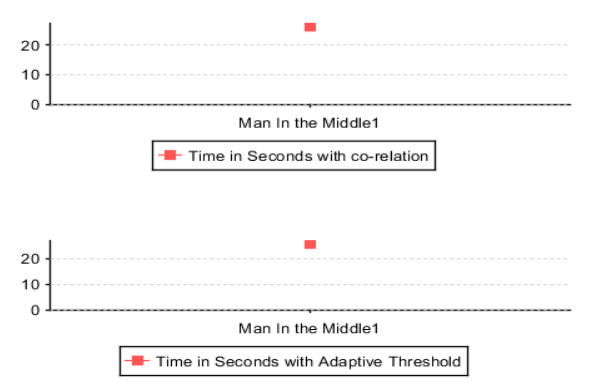

(d)

Fig. 4. (a) Media Transfer (b) Select File (c) Message From Attacker (d) Data Alteration Analysis

\section{IV.CONCLUSION}

This project aims to provide the security to agent or computer system. There are three Security Goals Are Confidentiality, Integrity, and Availability. Protect the confidentiality of data. Preserve the integrity of data. Promote the availability of data for authorized use. This application is also used to detect the attacks and provide prevention to system from strategic attacks.

\section{REFERENCE}

[1] Kumbhar Ankit Rajaram, Diwan Gaurav Prakash and Nikam D A.(2016).Consensus Problems in Networks of Agents with Switching Topology, (pg. 828- 830).

[2] Kumar Rajnish, Shahi Swati, (April 2013). Synchronization of coupled harmonic oscillators with 


\section{International Journal of Engineering Applied Sciences and Technology, 2019 \\ Vol. 3, Issue 12, ISSN No. 2455-2143, Pages 103-106 \\ Published Online April 2019 in IJEAST (http://www.ijeast.com)}

local interaction, International Journal of Engineering Trends and Technology (IJETT) ( pp. 1231-1236).

[3] R. Merris, (1994). Distributed secure coordinated control of multi agent systems. Laplacian matrices of a graph: a survey. Linear Algebra and its Applications,(197:143176).

[4] Feng Z., Sun C., and Hu G.,( 2016) Robust connectivity preserving rendezvous of multi-robot systems under unknown dynamics and disturbances, IEEE Trans. Control Netw. Syst, to be published, doi:10.1109.

[5] Dong Y. and Huang J.,(2013),A leader-following rendezvous problem of double integrator multi-agent systems," Automatica, vol. 49, no. 5,( pp. 1386-1391)

[6] Fragoso M. D. and Costa O. L. V.,(2005) A unified approach for stochastic and mean square stability of continuous-time linear systems with Markovian jumping parameters and additive disturbances," SIAM J. Control Optim., vol. 44, no. 4, (pp. 1165-1191).

[7] Olfati-Saber R. and Murray R. M.,(2004),Consensus problems in networks of agents with switching topology and time-delays, IEEE Trans. Autom. Control, vol. 49, no. 9, pp. (1520-1533)

[8] Ren W.,(2008), Synchronization of coupled harmonic oscillators with local interaction, Automatica, vol. 44, no. 12, (pp. 3195-3200)

[9] Wen G., Hu G., Yu W., and Chen G.,(2014),Distributed $\mathrm{H} \infty$ consensus of higher-order multiagent systems with switching topologies, IEEE Trans. Circuits Syst. II, Exp. Briefs, vol. 61, no. 5, (pp. 359-363)

[10] Hu G.,(2012), Robust consensus tracking of a class of second-order multiagent dynamic systems," Syst. Control Lett., vol. 61, no. 1, (pp. 134-142)

[11] Bini D. A., Iannazzoa B., and Poloni F., (2008),A fast Newton's method for anonsymmetric algebraic Riccati equation, SIAM J. Matrix Anal. Appl., vol. 30, no. 1, (pp. 276-290).

[12] Zhu Q. and Basar T.,( 2015), Game-theoretic methods for robustness, security, and resilience of cyberphysical control systems: Games-in-games principle for optimal cross-layer resilient control systems, IEEE Control Syst., vol. 35 , no. 1 , (pp. 46-65) 